\title{
Principales factores relacionados con las infecciones asociadas a la atención en salud en población neonatal entre 2014 a 2020. Revisión sistemática
}

\author{
Main factors related with the acquisition of health care \\ associated infections in the neonatal population between \\ 2014 to 2020. Systematic review
}

\author{
Kateryn Muñoz-Ante1, Christopher Ortega-Amaya', Jhonathan Wesley \\ Atencia-Poveda', María Carolina García-Restrepo1, Erika Francisca Garrido-Zea²
}

Resumen. Introducción. Las infecciones asociadas a la atención en salud son un efecto adverso frecuente. Los neonatos son un grupo poblacional afectado debido a su inmadurez inmunitaria. La Organización Mundial de la Salud estima que estas infecciones varían entre $5 \%$ a $25 \%$ en función del nivel de ingresos de cada país. En Colombia se evidencia un subregistro para estas infecciones, particularmente en los neonatos. Este estudio buscó identificar los factores de riesgo, microorganismos más comunes, y las vías de transmisión y colonización relacionadas a las infecciones asociadas a la atención en salud en neonatos, según lo reportado en la literatura mundial. Metodología. Se realizó una búsqueda sistemática por duplicado, que incluyó pesquisa de artículos, primera lectura y exclusión de duplicados, conciliación entre autores y evaluación de la calidad, en la base de datos PubMed, con artículos publicados entre los años 2014 a 2019. Resultados. El nivel de evidencia y el grado de recomendación fue bueno en general (niveles 1 y 2, recomendación A y B). Los factores de riesgo más comunes fueron la prematuridad y el bajo peso al nacer, el uso de catéter venoso central, ventilación mecánica, estancia hospitalaria prolongada y el uso de antibióticos por largo tiempo. Se reportaron microorganismos de importancia como Staphylococcus coagulasa negativos y Serratia marcenscens, y vías de transmisión y colonización comunes como el torrente sanguíneo, la vía respiratoria, la gastrointestinal y la piel. Conclusión. Las infecciones asociadas a la atención en salud son más frecuentes de lo estimado y

\footnotetext{
${ }^{1}$ Estudiantes del Programa de Medicina. Facultad de Ciencias de la Salud, Corporación Universitaria Remington. Medellín, Colombia.

2 Microbióloga y Bioanalista, MSc en Biología. Docente-Investigador, Líder Grupo de Investigaciones Biomédicas Uniremington, Facultad de Ciencias de la Salud, Corporación Universitaria Remington. Medellín, Colombia. E-mail: erika.garrido@ uniremington.edu.co.
}

Conflicto de interés: los autores declaran que no tienen conflicto de interés.

Medicina \& Laboratorio 2021;25:513-524. https://doi.org/10.36384/01232576.436.

Recibido el 12 de febrero de 2021; aceptado 29 de marzo de 2021. Editora Médica Colombiana S.A., 2021 
se pueden prevenir con la adopción de políticas sanitarias, como son un correcto lavado de manos, la desinfección del ambiente y equipos médicos, un adecuado cuidado de la piel del neonato y protocolos de aislamiento para pacientes con estas infecciones.

Palabras clave: infección hospitalaria, neonato, microorganismos, factores de riesgo, transmisión.

Abstract. Introduction. Health care-associated infections are a frequent adverse effect. Neonates are an affected population group due to their immune immaturity. The World Health Organization estimates that these infections vary between $5 \%$ to $25 \%$ depending on the income level of each country. In Colombia these infections are underreported, particularly in neonates. The aim of this review was to identify the risk factors, the most common microorganisms, and the routes of transmission for these type of infections in neonates, as reported in the world literature. Methodology. A systematic duplicate search was carried out in PubMed, with articles published between 2014 to 2019, which included a search for articles, reading and exclusion of duplicates, reconciliation between authors, and quality assessment. Results. The level of evidence and the grade of recommendation was optimal in general (levels 1 and 2, recommendation $A$ and $B$ ). The most common risk factors were prematurity and low birth weight, the use of central venous catheter, mechanical ventilation, prolonged hospital stay, and long-term use of antibiotics. Microorganisms such as coagulase negative Staphylococcus and Serratia marcenscens were reported, and common routes of transmission such as the bloodstream, respiratory tract, gastrointestinal tract, and skin were described. Conclusion. Health care-associated infections are more frequent than estimated, and can be prevented with the adoption of health policies, such as proper hand washing, disinfection of environmental surfaces and medical equipment, an adequate skin care of the neonate, and isolation protocols for patients with these infections.

Keywords: health care-associated infection, neonate, microorganism, risk factors, transmission.

\section{Introducción}

La Organización Mundial de la Salud (OMS) define las infecciones asociadas a la atención en salud (IAAS) o infecciones nosocomiales como "infecciones contraídas por un paciente durante su tratamiento en un hospital u otro centro sanitario, y que dicho paciente no tenía ni estaba incubando en el momento de su ingreso". Algunos estudios han mencionado un límite de tiempo de 48 a 72 horas después de ingresar a un centro asistencial de salud o hasta 30 días después de haber sido dado de alta [1].

Las IAAS afectan a todos los grupos etarios; sin embargo, su presentación tiende a ser más relevante en los neonatos por la inmadurez de su sistema de defensa [2], en pacientes con un sistema inmunitario afectado por alguna condición crónica (tuberculosis, infección por $\mathrm{VIH}$, cáncer, enfermedad renal crónica, etc.), y en pacientes geriátricos por las características propias 
del envejecimiento que disminuyen la funcionalidad de su sistema inmune frente a agentes externos [3]. Dentro de estas poblaciones, específicamente en la neonatal, se han encontrado algunas vías de trasmisión tales como el canal del parto, la lactancia, la transmisión transuterina, excesivas intervenciones manuales o con equipos médicos en el canal de parto, un lavado de manos inadecuado por el personal de salud [4], fallas en la esterilización de equipos médicos, y una inadecuada limpieza del ambiente del neonato [5]; a su vez, hay algunos microorganismos que bien pueden tener o no relación con las vías de transmisión, tales como Staphylococcus coagulasa negativos, Enterococcus, Staphylococcus aureus, Escherichia coli, Klebsiella pneumoniae, Pseudomonas aeruginosa, Acinetobacter baumanni, Sphingomonas paumobilis, Candida albicans y Candida spp. [3,6-9].

En la literatura se encuentran descritos factores que facilitan la adquisición y el desarrollo de las IAAS; entre ellos, bajo peso al nacer, parto prematuro y enfermedades concomitantes (infección intraamniótica, asfixia en el nacimiento, ruptura prematura de membranas), así como factores ambientales como la necesidad de procedimientos invasivos y el uso de sistemas de soporte vital (ventiladores, catéteres, etc.) $[3,6,10]$. Debido al subregistro de este tipo de infecciones, especialmente en neonatos, se desconoce la carga epidemiológica específica de las IAAS en este grupo de pacientes; sin embargo, se ha encontrado información respecto a su incidencia en la población general de países desarrollados, la cual varía entre 2,2 a 8,6 por cada 1.000 nacidos vivos [8]. La OMS estima que entre el $5 \%$ al $10 \%$ de los pacientes que son atendidos en instituciones de salud en países desarrollados contraerán una IAAS, mientras que en países en vías de desarrollo podría superar el $25 \%$ [8]. Adicionalmente, se ha reportado la prevalencia de las IAAS en algunos países como Mali (18,7\%), Brasil (14\%), Canadá $(11,6 \%)$, Inglaterra (9\%), España $(8,1 \%)$, Cuba (7,3\%), Indonesia $(7,1 \%)$ y Francia $(4,4 \%)[7,8]$.

A pesar de ser una problemática mundial, los datos exactos de mortalidad, especialmente en neonatos, también se desconocen. En la población en general, en Inglaterra se presentan más de 100.000 IAAS, las cuales generan más de 5.000 muertes. En Estados Unidos se estima que 1 de cada 136 pacientes hospitalizados se enferman gravemente debido a las IAAS, lo que causa más de 80.000 muertes por año, y en México se estiman 450.000 casos de IAAS que generan una tasa de 32 muertos por cada 100.000 habitantes [8]; adicionalmente, en este país la incidencia es aproximadamente de 4 a 15,4 casos de infección por cada 1.000 nacidos vivos, y de 8,8 a 41,1 por cada 100 egresos de $\mathrm{UCl}[7]$.

A pesar de que de las IAAS pueden considerarse el efecto adverso más común en la población neonatal con respecto a la atención sanitaria, y ser una causa importante de morbilidad y mortalidad principalmente por sepsis neonatal, no se cuenta con muchos estudios que brinden registros o datos epidemiológicos claros en Colombia [11]. Para la población general, las estadísticas revelaron un promedio histórico para el periodo 2002 a 2006, con una tasa de IAAS del 13,9\% por 1.000 días/estancia [7,8]. En 2013 hubo una proporción de infecciones asociadas a la asistencia sanitaria del 1,25\%, y se estimó que la incidencia de IAAS en las $\mathrm{UCl}$ varió entre $7 \%$ y $24,5 \%[7,12]$. En el caso específico de Bogotá, se encontraron mayores tasas de infección del torrente sanguíneo asociadas a catéter 
central, y de neumonía asociada al ventilador, tanto en unidades de adultos como en las pediátricas $[9,13]$.

Se pretende con esta revisión sistemática, conocer los factores, vías de colonización y transmisión de las IAAS, así como los principales microorganismos que hacen ineficaz su prevención en el neonato a nivel mundial.

\section{Metodología}

Revisión sistemática que se llevó a cabo mediante una búsqueda por duplicado, en la base de datos PubMed, de artículos publicados entre los años 2014 a 2019. Esta búsqueda se realizó dividiendo al equipo de investigadores en 2 grupos ( $A$ y B), de tal modo que se pudiera hacer una discriminación escrupulosa de toda la información encontrada en dichas fuentes.

La búsqueda y valoración de los hallazgos se realizó mediante 4 pasos: 1) pesquisa de los artículos en las bases de datos con el uso de términos $\mathrm{MeSH}$ (del inglés, Medical Subject Heading) "nosocomial", "infection", "neonate" y el operador booleano "and", donde los grupos A y B encontraron un total de 81 artículos; 2) lectura del título y resumen de cada documento con eliminación de los duplicados, donde se excluyeron 13 artículos, el grupo A aprobó 32 artículos y el grupo B 29 artículos, para un total de 61 artículos; 3) se hizo una conciliación entre los investigadores respecto a los 7 artículos en los que hubo discordancia, y así llegar a un resultado aceptado por todos, ambos grupos aprobaron 26 artículos y denegaron 42 artículos, por no cumplir con los criterios de inclusión (tabla 1); y, 4) se evaluó la calidad de los artículos aceptados, y se descartaron los que no fueron de alta calidad, para un total de 9 artículos aprobados para usar como material bibliográfico de la investigación (figura 1).

\section{Evaluación de calidad de los artículos}

Para la evaluación de la calidad de los artículos se tuvo en cuenta el grado de recomendación y el nivel de evidencia del Centre for Evidence-Based Medicine [14], considerando los siguientes aspectos: una introducción que incluyera una mención concisa de la problemática a tratar en el estudio, dando una base para la elaboración del artículo; una metodología que describiera la población de estudio, tiempo en el que se realizó, instrumentos que se usaron para la recolección o manejo de los datos, el paso a paso del desarrollo de

\begin{tabular}{l}
\hline Tabla 1. Criterios de inclusión y exclusión \\
\hline Criterios de inclusión \\
\hline Artículos publicados entre el periodo 2014 a \\
2019 \\
Artículos en inglés \\
Artículos científicos originales, revisiones \\
sistemáticas, metaanálisis, ensayos aleatorios \\
controlados relacionados con infecciones \\
nosocomiales en neonatos \\
Artículos que incluyeran la necesidad de \\
intervención médico-instrumental, medidas \\
de bioseguridad en los trabajadores de salud, \\
y medidas de higiene intrahospitalarias \\
\hline Criterios de exclusión
\end{tabular}

Abstracts (resúmenes de artículos no abiertos al público)

Reporte de casos

Capítulos de libros

Resúmenes de conferencias

Guías de buena práctica

Artículos que describieran las infecciones nosocomiales sin establecer las causas principales para su desarrollo en los neonatos

Manuales e instructivos 


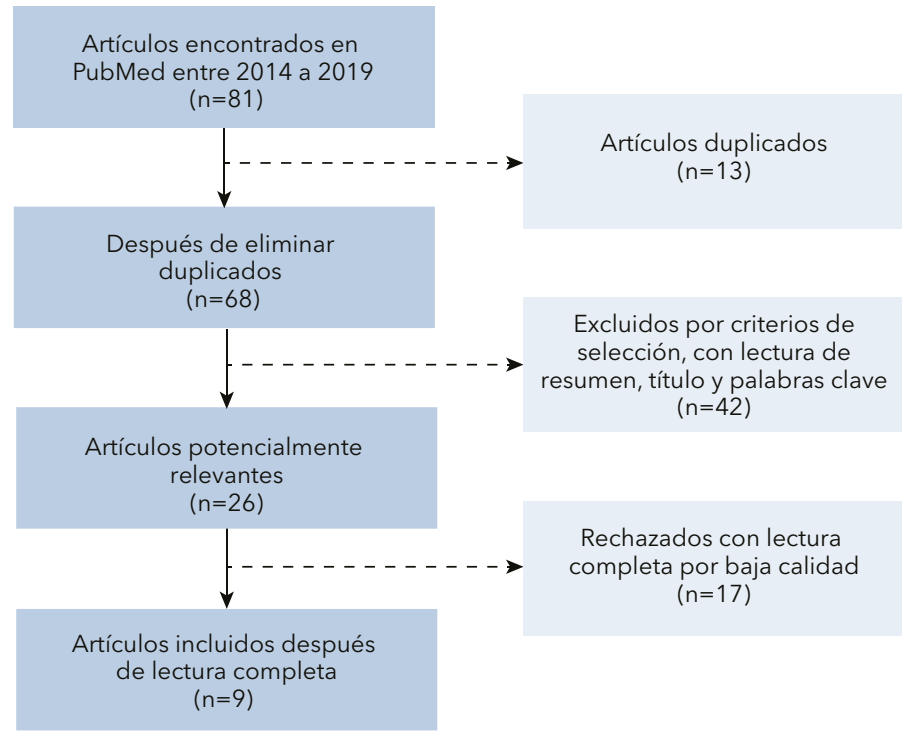

Figura 1. Proceso de selección de los artículos.

la investigación, intervenciones realizadas y la presencia de tablas o gráficas; unos resultados que tuvieran coherencia con los objetivos planteados, que fueran claros, con presencia de gráficas o tablas, y con hallazgos incidentales que pudieran haber afectado la investigación; una discusión sin presencia de conflictos de interés o que se hubieran resuelto por los autores, con mención de limitaciones en la investigación y descripción de estas, y presencia de posibles sesgos; finalmente, una conclusión que tuviera una redacción clara, coherencia con los objetivos y resultados planteados, y si estos últimos fueron los esperados (tabla 2).

\section{Resultados}

De los artículos seleccionados, dos mencionaban la sepsis neonatal de desarrollo tardío (LOS, del inglés, La-

Tabla 2. Nivel de evidencia y grado de recomendación de los artículos

\begin{tabular}{lcc}
\hline Artículos & Nivel de evidencia & Grado de recomendación \\
\hline Dong Y et al [15] & $2 b$ & $\mathrm{~B}$ \\
\hline Darmstadt GL et al [22] & $1 \mathrm{c}$ & $\mathrm{A}-\mathrm{B}$ \\
\hline Cristina ML et al [23] & $2 \mathrm{c}$ & $\mathrm{B}$ \\
Cailes B et al [16] & $2 \mathrm{~b}$ & $\mathrm{~B}$ \\
\hline Stapleton PJ et al [21] & $1 \mathrm{a}$ & $\mathrm{A}$ \\
Hartz LE et al [18] & $1 \mathrm{~b}$ & $\mathrm{~A}-\mathrm{B}$ \\
Moffa M et al [19] & $1 \mathrm{a}$ & $\mathrm{A}$ \\
\hline Ramasethu J et al [20] & $2 \mathrm{a}$ & $\mathrm{B}$ \\
Wang Let al [17] & $1 \mathrm{a}$ & $\mathrm{A}$ \\
\hline
\end{tabular}


te-Onset Sepsis) como uno de los desenlaces de la transmisión de las IAAS $[15,16]$; los factores de riesgo más comunes para LOS fueron el uso de catéter venoso central $[16,17]$, el uso de ventilación mecánica [17-20], la estancia hospitalaria prolongada, y el uso de antibióticos de forma prolongada $[15,16,18,21]$. En cinco artículos, la prematuridad y el bajo peso al nacer fueron los principales factores de riesgo para IAAS $[17,19,20,22,23]$. En tres artículos revisados se menciona a Serratia marcescens como uno de los microorganismos más comunes asociados a las IAAS. En la unidad de cuidados intensivos neonatales, este microorganismo se ha detectado principalmente en superficies de lavabos, sistemas de aire acondicionado, broncoscopios, laringoscopios, nebulizadores, equipos de ventilación, dispensadores de soluciones desinfectantes (jabón, gel antibacterial), y en la nutrición parenteral contaminada, entre otros $[17,21,23]$. En tres artículos que estudiaron las rutas de transmisión, se menciona la piel como la vía más susceptible a cambios y/o deterioro $[15,18,22]$, y en otros el torrente sanguíneo como la ruta de colonización y transmisión más frecuente $[16,20,22,23]$. En la tabla 3 se presentan los principales hallazgos de los estudios realizados en diferentes regiones del mundo, donde se muestran aspectos generales de las IAAS.

En la tabla 4 se describen los factores de riesgo más comunes, las principales vías de transmisión y lugares de colonización, así como los microorganismos más frecuentemente hallados. Se dificultó la expresión de frecuencias de estos, debido a que fueron específicos e individuales de cada país e incluso de cada centro hospitalario, por lo cual no se pudo llegar a una frecuencia de presentación en común en un contexto general.

\section{Discusión}

Las IAAS se han establecido como uno de los eventos adversos más comunes relacionados a la atención en salud; sin embargo, es muy poca la información que se recolecta de este fenómeno, especialmente en países en vías de desarrollo. En México, la Red Hospitalaria de Vigilancia Epidemiológica (RHOVE) reportó que en 2015 las infecciones del torrente sanguíneo relacionadas a catéter venoso central o catéter venoso periférico, representaron un factor de riesgo para desarrollar IAAS en un $14 \%$ y $13,2 \%$, respectivamente, las cuales asociadas a la contaminación de soluciones y a las infecciones secundarias a procedimientos, ocuparon el primer lugar de las IAAS, y los más afectados por dichas infecciones fueron los menores de 5 años, particularmente los menores de 1 mes. Además, se destaca que los servicios donde se reportó mayor distribución de infecciones fueron Pediatría y la UCIN [24]. De igual forma, en Estados Unidos y Europa, las infecciones del torrente sanguíneo son las más comunes en la UCIN; la neumonía asociada al respirador, la peritonitis, la conjuntivitis y los abscesos cutáneos son importantes, pero menos comunes [25]. La información encontrada en la literatura seleccionada muestra que las IAAS se presentan por diferentes factores de riesgo, pero principalmente por las características propias del neonato, como prematuridad [19,20,22,23], bajo o muy bajo peso al nacer $[17,20,23]$, así como por factores asociados con la atención del neonato, incluyendo el uso del catéter venoso central $[16,17]$, la ventilación mecánica invasiva [17-19] y el contacto con las superficies hospitalarias [18].

También se encontraron factores como los descritos por Morales-Alvarez y colaboradores [24], tales como la sobreu- 
Principales factores relacionados con las infecciones asociadas a la atención en salud en población neonatal entre 2014 a 2020. Revisión sistemática

Tabla 3. Resultados por estudio

\begin{tabular}{|c|c|c|c|}
\hline Autores & Año & Región & Hallazgos \\
\hline Dong Y et al [15] & 2014 & $\begin{array}{c}\text { Asia, América, } \\
\text { Australia, } \\
\text { Europa }\end{array}$ & $\begin{array}{l}\text { Staphylococcus epidermidis responsable de más } \\
\text { del } 50 \% \text { de sepsis de origen tardío en países } \\
\text { industrializados. Factores de riesgo: estancia } \\
\text { hospitalaria prolongada, procedimientos invasivos } \\
\text { repetidos y prolongados }\end{array}$ \\
\hline $\begin{array}{l}\text { Darmstadt GL et } \\
\text { al [22] }\end{array}$ & 2014 & Asia & $\begin{array}{l}\text { La prematuridad hace susceptible a los bebés a } \\
\text { infecciones graves de piel }\end{array}$ \\
\hline $\begin{array}{l}\text { Cristina ML et } \\
\text { al [23] }\end{array}$ & 2019 & Europa & $\begin{array}{l}\text { Serratia marcescens se encuentra más } \\
\text { frecuentemente en la sangre, seguida por el aparato } \\
\text { respiratorio y tracto gastrointestinal. En los recién } \\
\text { nacidos, los principales factores de riesgo son: } \\
\text { inmadurez del sistema inmune y bajo peso }(<1.500 \\
\text { g) }\end{array}$ \\
\hline Cailes B et al [16] & 2015 & Australia & $\begin{array}{l}\text { Las infecciones de la sangre se asocian entre } 1 \% \text { a } \\
20 \% \text { con el uso de catéteres venosos centrales }\end{array}$ \\
\hline $\begin{array}{l}\text { Stapleton PJ et } \\
\text { al [21] }\end{array}$ & 2015 & Europa & $\begin{array}{l}\text { Los factores de riesgo encontrados fueron: alto } \\
\text { consumo antibiótico, brote previo en la UCIN, } \\
\text { control de infecciones deficientes y hacinamiento. } \\
\text { Los microorganismo causantes en los estudios } \\
\text { fueron Klebsiella spp., Enterobacter spp., Escherichia } \\
\text { coli, Salmonella spp., Serratia marcescens }\end{array}$ \\
\hline $\begin{array}{l}\text { Hartz LE et al } \\
{[18]}\end{array}$ & 2015 & América & $\begin{array}{l}\text { En las UCIN existe un riesgo de infección por } \\
\text { parte del neonato del 55,9\% para Staphylococcus } \\
\text { coagulasa negativa. Factores asociados a los } \\
\text { cambios en el microbioma del bebé: contacto con } \\
\text { los padres, alimentación, superficies, espacios de } \\
\text { trabajo de enfermería, cuidado infantil (incubadoras, } \\
\text { ventiladores, calentadores), personal de salud, piel } \\
\text { contaminada de los cuidadores y antibióticos }\end{array}$ \\
\hline
\end{tabular}

Se encontró que los bebés prematuros, con bajo peso al nacer o que requirieron el uso de sistemas de soporte vital, que hubiesen estado expuestos

Moffa M et al [19]

2017

América

Ramasethu J et al [20]

2017

América

Wang Let al [17] 2019 a agua de grifo, ventiladores e incubadoras, entre otros, presentaban riesgo de infección por microorganismos como Flavobacterium meningosepticum, Enterobacter cloacae, Pseudomonas aeruginosa, Klebsiella pneumoniae y Staphylococcus coagulasa negativa

La prematuridad y el bajo peso al nacer hacen susceptible a los neonatos para la colonización de microorganismos como Staphylococcus coagulasa negativa, Staphylococcus aureus, Enterococcus, Streptococcus grupo B, Enterobacter, Klebsiella, Escherichia coli, Pseudomonas, Candida albicans, Candida parapsilosis, virus respiratorio sincitial, rinovirus, influenza

Los factores de riesgo encontrados en este estudio fueron: bajo peso al nacer $(<2.500 \mathrm{~g})$, edad gestacional (<37 semanas), punción venosa, asfixia, intolerancia a la alimentación y uso de ventilación mecánica 
Tabla 4. Factores de riesgo, vías de transmisión y colonización, y microorganismos comunes

\begin{tabular}{l}
\hline Factores de riesgo \\
\hline Bajo peso al nacer $(<2.500 \mathrm{~g})$ \\
Muy bajo peso al nacer $(<1.500 \mathrm{~g})$ \\
Prematuridad (<37 semanas) \\
Ventilación mecánica \\
Catéter venoso central \\
Pobre higiene de manos \\
Uso prolongado de antibióticos \\
Equipos médicos (incubadoras, calentadores) \\
\hline Vías de transmisión y sitio de colonización \\
\hline Sanguínea \\
Respiratoria \\
Gastrointestinal \\
\hline Piel \\
\hline Microorganismos \\
\hline
\end{tabular}

Staphylococcus epidermidis

Serratia marcescens

Klebsiella pneumoniae

Escherichia coli

Pseudomonas aeruginosa

Staphylococcus aureus

Virus sincitial respiratorio

Rinovirus

tilización de antibióticos [15,18,21], las visitas de los familiares y la insuficiencia de personal sanitario [21], que hacen difícil seguir los protocolos de limpieza; además del lavado y desinfección inadecuados de las manos como vehículo de contaminación de la piel y/o mucosas del recién nacido, que favorecen la permanencia y difusión de bacterias patógenas [18-20,23].

Según lo reportado en Estados Unidos y Europa, los microorganismos grampositivos como Staphylococcus coagulasa negativos y Staphylococcus aureus son la causa predominante de sepsis de aparición tardía en la UCIN (48\% a $70 \%$ de los casos); en segundo lugar, los microorganismos gramnegativos como Serratia marcescens y Pseudomonas aeruginosa (19\% a 25\% de los casos); y, en tercer lugar, pero no menos importantes, los hongos como Candida albicans (12\% a $18 \%$ de los casos) $[15,16,18-21,23,25]$. Esto coincide con lo descrito en México, donde los Staphylococcus coagulasa negativos son los microorganismos más frecuentemente aislados en recién nacidos de muy bajo peso al nacer [24], y lo reportado en los resultados de la revisión a nivel de América, Europa y Asia [18-21,23], donde además se encontró asociación con otros agentes como Staphylococcus epidermidis [15], Enterococcus spp. [20], Streptococcus grupo B [20], Escherichia coli [20,21], Enterobacter cloacae [19,20], KlebsieIla pneumoniae $[19,21$ y algunos virus como el sincitial respiratorio y el rinovirus [20], siendo estos últimos reportados como causas de epidemia en las UCIN [26], pero su verdadera incidencia a este nivel es sobreestimada [13].

Con respecto a las vías o rutas de transmisión y colonización para el desarrollo de IAAS en neonatos, la principal fue el torrente sanguíneo [16,20,22,23], muy similar a lo reportado por otros autores como Karlowicz y colaboradores [27], quienes informaron que las infecciones del torrente sanguíneo fulminantes de inicio tardío fueron las más frecuentes (69\%), causadas por organismos gramnegativos en su mayoría. De manera similar, Scamardo y colaboradores [28] reportaron que la infección del torrente sanguíneo asociada a la vía central fue la infección más frecuente en el 69,6\% de los casos, causada principalmente por Klebsiella pneumoniae. Entre otras vías, se menciona la respiratoria en segundo 
lugar de frecuencia (28\%), representada principalmente por neumonía en los infantes con bajo peso y ventilación mecánica $[20,23,25,28]$, de donde se aislaron bacterias gramnegativas de los aspirados traqueales, aunque en su mayoría, los casos fueron polimicrobianos $[23,25]$. La vía de infección gastrointestinal es el tercer lugar de colonización y transmisión más frecuente, especialmente por enterobacterias como Serratia marcescens [23]. $\mathrm{Se}$ ha reportado a nivel mundial que las enterobacterias son responsables del $75 \%$ de los casos de peritonitis asociada a enterocolitis necrotizante y del $25 \%$ de los casos de peritonitis por perforación intestinal. Candida albicans y Staphylococcus coagulasa negativos también se han aislado, pero en menor proporción [25]. Con relación a la piel, se encontró que al igual que lo descrito por Morales-Alvarez y colaboradores [24], los recién nacidos pretérmino en cuidados intensivos son más susceptibles a la sepsis adquirida por la piel, debido a que sufren exposición a diferentes factores como la falta de desarrollo de las capas cutáneas y déficit de vérnix; adicionalmente, el contacto con superficies, con el personal de salud o los propios padres o familiares, y la falta de aplicación de sustancias tipo emolientes, hacen esta vía más susceptible a cambios y/o deterioro [16,18,22]. La mayoría de las infecciones neonatales por piel como vía de entrada, son causadas por Staphylococcus aureus, produciendo manifestaciones clínicas como impétigo, celulitis, abscesos de tejidos blandos, piel escaldada, y síndromes de choque tóxico, principalmente en infantes de bajo peso $(<1.000 \mathrm{~g})$ [25].

De los microorganimos descritos, merece especial mención Serratia marcescens como uno de los microorganimos más comúnmente asociados a las IAAS [29,30], ya que representa el $15 \%$ de todos los aislamientos de infecciones nosocomiales. Este microorganismo puede llegar a colonizar pacientes neonatales de forma asintomática o en forma de manifestaciones clínicas, tales como la queratitis, conjuntivitis, infecciones del tracto urinario, de sitios quirúrgicos, del torrente sanguíneo, o neumonía, meningitis y sepsis $[19,21,23]$. El sitio de infección más frecuente por este microorganismo es el torrente sanguíneo, seguido por el aparato respiratorio y el tracto gastrointestinal. Esta especie muestra resistencia intrínseca a varias clases de antibióticos, incluidos algunos betalactámicos y tetraciclinas. El principal factor de riesgo para el desarrollo de sepsis y bacteriemia por Serratia marcescens es la hospitalización [21,23].

\section{Conclusión}

Teniendo en cuenta los factores de riesgo mencionados para el desarrollo de IAAS en la población neonatal, se puede concluir que este evento adverso se da como resultado de políticas de prevención deficientes y de la poca importancia que se les da, lo que dificulta la recolección de información con el fin de desarrollar mecanismos preventivos. Un punto clave para subsanar el desarrollo de estas infecciones es mejorar la atención médico-paciente, con el fin de evitar procedimientos y elementos médicos invasivos innecesarios, mejorar las labores de asepsia y antisepsia, tanto de los procedimientos médicos como de las superficies hospitalarias, así como del personal al cuidado de los neonatos; también, proporcionar un aislamiento adecuado para aquellos neonatos que hayan desarrollado una IAAS, con el fin de prevenir que esta se expanda a otros servicios hospitalarios y a otros pacientes. 
Se deben establecer acciones positivas como evitar aglomeraciones del personal en las UCIN, el correcto lavado de manos, desinfección de las superficies y entorno hospitalario y el uso obligatorio de material invasivo nuevo (en el caso de sondas o catéteres), así como la esterilización adecuada de equipos médicos, el cuidado de la piel del neonato, desinfección del sitio de punción para los catéteres, un buen manejo de antibióticos, inicio de alimentación enteral de forma rápida y adecuada, y el aislamiento de neonatos con infecciones diagnosticadas. También se deben seguir políticas sanitarias de prevención que impacten estas infecciones, las cuales tendrían que ser de conocimiento público y obligatorio para el personal de salud y familiares, dirigidas siempre a mejorar la técnica aséptica con mejores resultados $[16,18,21,23]$.

\section{Agradecimientos}

El grupo investigador quiere agradecer a la Corporación Universitaria Remington por brindar los recursos, las herramientas y los tutores necesarios para llevar a cabo el proceso de investigación, y poder culminar este proyecto.

\section{Referencias}

1. Organización Mundial de la Salud (OMS). Carga mundial de infecciones asociadas a la atención sanitaria. Ginebra, Suiza: OMS; 2018. Acceso 28 de noviembre de 2020. Disponible en https://www.who.int/gpsc/country_work/burden_hcai/es/.

2. Heath JA, Zerr DM. Chapter 35 - Infections acquired in the nursery: Epidemiology and control. In: Remington JS, Klein JO, Wilson CB, Baker CJ, eds. Infectious Diseases of the Fetus and Newborn Infant (Sixth Edition). Philadelphia: W.B. Saunders; 2006. p. 1179-1205.
https://doi.org/https://doi.org/10.1016/B0-72160537-0/50037-2.

3. Vergnano S, Heath PT. Fetal and neonatal infections. Medicine 2017;45:715-722. https:// doi.org/10.1016/j.mpmed.2017.08.011.

4. Mariani M, Bandettini R, La Masa D, Minghetti D, Baldelli I, Serveli S, et al. Bacterial invasive infections in a neonatal intensive care unit: a 13 years microbiological report from an Italian tertiary care centre. J Prev Med Hyg 2020;61:e162166. https://doi.org/10.15167/2421-4248/ jpmh2020.61.2.1401.

5. Hooven TA, Polin RA. Healthcare-associated infections in the hospitalized neonate: a review. Early Hum Dev 2014;90:s4-6. https://doi. org/10.1016/s0378-3782(14)70002-7.

6. Avila-Puentes MA, Triana-Trujillo LM. Boletín epidemiológico de infecciones asociadas a la atencion en salud (IAAS) y resistencia bacteriana 2015. Bogotá D.C.: Alcaldía Mayor de Bogotá; 2015. Acceso 19 de octubre de 2020. Disponible en http://www.saludcapital.gov.co/DSP/Infecciones\%20Asociadas\%20a\%20Atencin\%20en\%20 Salud/Boletines/BOLETIN_IAAS_2015.pdf.

7. García H, Martínez-Muñoz ÁN, PeregrinoBejarano L. Epidemiología de las infecciones nosocomiales en una unidad de cuidados intensivos neonatales. Rev Med Inst Mex Seguro Soc 2014; 52:30-37.

8. Gaviria-Uribe A, Ruiz-Gómez F, Muñoz-Muñoz N, Burgos-Bernal G, Arias-Duarte J, García-De Vargas S. Detectar, prevenir y reducir infecciones asociadas con la atención en salud. Bogotá D.C.: Ministerio de Salud y Protección Social; 2015. Acceso 20 de noviembre de 2020. Disponible en https://www.minsalud.gov.co/ sites/rid/Lists/BibliotecaDigital/RIDE/DE/CA/ Detectar-Infecciones.pdf.

9. Secretaría Distrital de Salud de Bogotá. Guía de prevención, vigilancia epidemiológica y control de las infecciones asociadas al cuidado de la salud en las unidades de recien nacidos. Bogotá D.C.: Secretaría Distrital de Salud de Bogotá D.C.; 2011. Acceso 02 de diciembre de 2020. Disponible en http://www.saludcapital.gov. co/sitios/VigilanciaSaludPublica/Todo\%20IIH/ Gu\%C3\%ADa\%20Recien\%20Nacido\%20pdf.pdf.

10. Khan AM, Morris SK, Bhutta ZA. Neonatal and perinatal infections. Pediatr Clin North Am 
2017;64:785-798. https://doi.org/10.1016/j. pcl.2017.03.008.

11. García H, Cervantes-Luna B, González-Cabello $\mathbf{H}$, Miranda-Novales G. Risk factors for nosocomial infections after cardiac surgery in newborns with congenital heart disease. Pediatr Neonatol 2018;59:404-409. https://doi. org/10.1016/j.pedneo.2017.11.014.

12. Allegranzi B, Bagheri-Nejad $S$, Garcia-CastiIlejos G, Kilpatrick C, Kelley E. Report on the burden of endemic health care-associated infection worldwide clean care is safer care. Geneva, Switzerland: World Health Organization; 2011. Acceso 15 de diciembre de 2020. Disponible en https://www.ncbi.nlm.nih.gov/books/ NBK144030/.

13. Herrera-Hernández JC. Caracterización de los pacientes con infección asociada a la atención en la salud (IAAS) en los servicios de cuidado intensivo y cuidado intermedio neonatal de la fundación HOMI Hospital de La Misericordia de Bogotá. Bogotá D.C.: Universidad Nacional de Colombia; 2018. Acceso 18 de diciembre de 2020. Disponible en https://repositorio.unal.edu.co/bitstream/handle/unal/63482/79916094.2018. pdf?sequence $=1$ \&isAllowed $=y$.

14. Burns PB, Rohrich RJ, Chung KC. The levels of evidence and their role in evidence-based medicine. Plast Reconstr Surg 2011;128:305-310. https://doi.org/10.1097/ PRS.0b013e318219c171.

15. Dong Y, Speer CP. The role of Staphylococcus epidermidis in neonatal sepsis: guarding angel or pathogenic devil? Int J Med Microbiol 2014;304:513-520. https://doi.org/10.1016/j. ijmm.2014.04.013.

16. Cailes B, Vergnano S, Kortsalioudaki C, Heath P, Sharland $\mathbf{M}$. The current and future roles of neonatal infection surveillance programmes in combating antimicrobial resistance. Early Hum Dev 2015;91:613-618. https:// doi.org/10.1016/j.earlhumdev.2015.08.012.

17. Wang L, Du KN, Zhao YL, Yu YJ, Sun L, Jiang HB. Risk factors of nosocomial infection for infants in neonatal intensive care units: A systematic review and meta-analysis. Med Sci Monit 2019;25:8213-8220. https://doi.org/10.12659/ MSM. 917185.
18. Hartz LE, Bradshaw W, Brandon DH, Gregory

KE. Potential NICU environmental influences on the neonate's microbiome: A systematic review. Adv Neonatal Care 2015;15:324-335. https:// doi.org/10.1097/anc.0000000000000220.

19. Moffa M, Guo W, Li T, Cronk R, Abebe LS, Bartram J. A systematic review of nosocomial waterborne infections in neonates and mothers. Int J Hyg Environ Health 2017;220:1199-1206. https://doi.org/10.1016/j.ijheh.2017.07.011.

20. Ramasethu J. Prevention and treatment of neonatal nosocomial infections. Matern Health Neonatol Perinatol 2017;3:5. https://doi. org/10.1186/s40748-017-0043-3.

21. Stapleton $P J$, Murphy $M$, McCallion N, Brennan M, Cunney R, Drew RJ. Outbreaks of extended spectrum beta-lactamase-producing Enterobacteriaceae in neonatal intensive care units: a systematic review. Arch Dis Child Fetal Neonatal Ed 2016;101:F72-78. https://doi. org/10.1136/archdischild-2015-308707.

22. Darmstadt GL, Ahmed $S$, Ahmed AS, Saha SK. Mechanism for prevention of infection in preterm neonates by topical emollients: a randomized, controlled clinical trial. Pediatr Infect Dis J 2014;33:1124-1127. https://doi. org/10.1097/inf.0000000000000423.

23. Cristina ML, Sartini M, Spagnolo AM. Serratia marcescens infections in neonatal intensive care units (NICUs). Int J Environ Res Public Health 2019;16:610. https://doi.org/10.3390/ ijerph16040610.

24. Morales-Álvarez CT, Cárdenas-Rodríguez ML, Moreno-González MM, Herrera-Paredes

JM. Neonato con terapia intravenosa: una revisión de la literatura dirigida a la prevencion de riesgos. Sanus 2020;5:1-14. https://doi. org/10.36789/sanus.vi13.151.

25. Sass L, Karlowicz MG. 94 - Healthcare-associated infections in the neonate. In: Long SS, Prober CG, Fischer M, eds. Principles and Practice of Pediatric Infectious Diseases (Fifth edition): Elsevier; 2018. p. 560-566. https://doi.org/ https://doi.org/10.1016/B978-0-323-401814.00094-3.

26. Vain NE. Nosocomial respiratory viral infection in the neonatal intensive care unit. Am J Perinatol 2020;37:s22-25. https://doi. org/10.1055/s-0040-1714081. 
27. Karlowicz MG, Buescher ES, Surka AE. Fulminant late-onset sepsis in a neonatal intensive care unit, 1988-1997, and the impact of avoiding empiric vancomycin therapy. Pediatrics 2000;106:1387-1390. https://doi.org/10.1542/ peds.106.6.1387.

28. Scamardo MS, Dolce P, Esposito EP, Raimondi F, Triassi M, Zarrilli R. Trends, risk factors and outcomes of healthcare-associated infections in a neonatal intensive care unit in Italy during 2013-2017. Ital J Pediatr 2020;46:34-34. https://doi.org/10.1186/s13052-020-0799-3.
29. Gastmeier P, Loui A, Stamm-Balderjahn S, Hansen S, Zuschneid I, Sohr D, et al. Outbreaks in neonatal intensive care units They are not like others. Am J Infect Control 2007;35:172-176. https://doi.org/10.1016/j. ajic.2006.07.007.

30. Raymond J, Aujard Y. Nosocomial infections in pediatric patients: a European, multicenter prospective study. European Study Group. Infect Control Hosp Epidemiol 2000;21:260-263. https://doi.org/10.1086/501755. 